\title{
BUENAS PRÁCTICAS EDUCATIVAS PARA EL APRENDIZAJE DE LA LENGUA INGLESA: ASPECTOS PEDAGÓGICOS
}

\author{
María Dolores Corpas Arellano \\ Universidad de Granada
}

RESUMEN: Este estudio empírico indaga sobre las prácticas pedagógicas utilizadas en el proceso de enseñanza-aprendizaje del inglés como lengua extranjera, su frecuencia y su repercusión en el rendimiento académico del estudiante de cuarto de ESO (Educación Secundaria Obligatoria) en la asignatura de inglés. El objetivo de esta investigación es conocer qué prácticas pedagógicas son las más adecuadas para el aprendizaje de la lengua inglesa y qué frecuencia deben tener en el aula.

Esta investigación concluye afirmando que las prácticas pedagógicas que favorecen, más fácilmente, el aprendizaje de la lengua inglesa son: el uso de materiales impresos editados, el uso del casete/ reproductor de CDs, la utilización del libro de texto y que la duración de los deberes sea breve.

PALABRAS CLAVE: Buenas prácticas, aprendizaje, enseñanza, inglés, ESO.

\section{BEST EDUCATIONAL PRACTICES TO LEARN ENGLISH: PEDAGOGIC ASPECTS}

\begin{abstract}
This empirical research investigates the pedagogical practices in the teaching-learning process of English as a foreign language, the frequency of these practices and their impact in $4^{\text {th }}$ year of Compulsory Secondary Education (CSE) students marks in English as a school subject. This study aims to know which pedagogical practices are the best to learn English and how often they should be used in the English language classroom.

This research concludes affirming that the best practices to learn English as a foreign language are: the use of printed materials, cassette/CDs players, textbooks, and finally, the duration of students English homework should be short.
\end{abstract}

KEYWORDS: Best practices, learning, teaching, English, CSE.

Recibido: 26/09/2012

Aceptado: 05/11/2012 


\section{INTRODUCCIÓN}

La búsqueda de acciones de mejora de la práctica docente ha sido constante a lo largo de la historia. El uso de métodos más innovadores y eficaces en el aprendizaje se encamina a la consecución de resultados más satisfactorios en un menor plazo de tiempo. Esta búsqueda de acciones de mejora de resultados no es algo exclusivo de la educación sino que son propios a cualquier actividad humana. La utilización reflexiva de propuestas pedagógicas creadas por profesorado que interaccione con las mismas es fundamental para el desarrollo de la práctica docente, según Alexander (1997).

Siguiendo esta línea de reflexión sobre las propuestas pedagógicas, nuestra investigación tiene como objetivo conocer y valorar las prácticas pedagógicas en el proceso de enseñanza-aprendizaje de la lengua inglesa, que se dan tanto dentro como fuera del aula, su frecuencia y su repercusión en la calificación media de los estudiantes. El objetivo último de esta investigación es conocer qué prácticas son más adecuadas para la adquisición de la lengua inglesa conforme a las características singulares del alumnado de cuarto de Educación Secundaria Obligatoria.

Los ítems incluidos en aspectos pedagógicos son los siguientes: el profesor imparte toda la clase en inglés, el profesor nos anima a comunicarnos en inglés, en clase de inglés usamos medios audiovisuales, en clase de inglés usamos el casete, en clase de inglés usamos el libro de texto, el profesor manda deberes y tiempo dedicado a los deberes.

\section{Concepto de buenas prácticas educativas}

\subsection{Origen del término "buenas prácticas"}

Si bien el término de buenas prácticas procede del mundo de la economía y de los negocios, desde los años noventa, se ha ido extendiendo a otros sectores como la política social y educativa. En economía, existen técnicas como benchmarking, indicadores de eficacia y competitividad y realización de comparaciones entre empresas a fin de incrementar los procesos de innovación corporativa (Brannan y otros, 2006).

En 1996, en la Cumbre de las Ciudades organizada por Naciones Unidas, se presentaron iniciativas para mejorar las condiciones de vida en las ciudades y políticas de desarrollo sostenible. A raíz de esta conferencia, se creó el Programa de Buenas Prácticas.

Poco tiempo después, Bolívar (1999), utiliza este término relacionado con la educación. Las buenas prácticas educativas surgen como prácticas relativas a las propuestas presentadas como ejemplos de buena enseñanza y aprendizaje, centros y docentes buenos y eficaces, además de, buenas políticas de gobierno, recursos, condiciones y procesos que faciliten las tareas en educación.

\subsection{Concepto de buenas prácticas en educación}

Vivimos en una sociedad en constante evolución. Transformaciones económicas, sociales y tecnológicas exigen que los individuos desarrollen una alta capacidad de adaptación a nuevas circunstancias y de resolución de problemas. La educación, 
como parte de la sociedad, no es ajena a estas transformaciones. En estos tiempos de expansión de la información y del conocimiento, se hace indispensable la creación del propio conocimiento del individuo. Para lograr este objetivo, parece sensato el acceso y la utilización juiciosa de buenas experiencias y prácticas realizadas por otros. Hargreaves (2003) opina a este respecto que la creación, el acceso y la gestión del conocimiento es un trayecto inexcusable a recorrer para crear y sostener organizaciones, actuaciones profesionales y políticas inteligentes al servicio de propósitos morales y justos.

Así, se buscan técnicas innovadoras que permitan una mejora de las prácticas educativas mediante el intercambio de modelos y experiencias educativas avaladas por sus buenos resultados, a pesar de que la transferencia de una situación de aprendizaje a otra siempre ha de ser adaptada al nuevo contexto. Como declaran Coffield y Edgard (2009: 238), "una buena práctica, nunca puede ser única, fija o abstracta, ni una predeterminación impuesta por alguien desde algún lugar o posición". A esto añaden que se trata de una experiencia o cultura escolar compartida. De hecho, una práctica puede ser buena para ciertos alumnos y no para otros. Se puede adaptar en determinados contextos pero no alcanzar sus objetivos en contextos distintos.

Otros autores como Abdoulaye (2003) y Coffield y Edgard (2009), denominan buenas prácticas de aprendizaje a aquellas estrategias de aprendizaje y procesos dirigidos a reducir las desigualdades entre el alumnado y a fomentar la equidad entre los individuos y grupos. Estos autores parten de la idea de que la eficacia de los métodos y de las acciones educativas no afecta de igual manera a todo el alumnado. Es indiscutible que hay grupos de alumnos que progresan más rápidamente que otros. A esto han Ilamado eficacia diferencial.

Escudero (2009), considera que una práctica considerada como buena es una realidad compuesta por valores, concepciones y propósitos, modos de hacer y formas de pensar, conocimientos explícitos e implícitos sostenidos por los prácticos, componentes racionales y otros que anidan en las vivencias, sentimientos y compromisos, aspiraciones y sueños, al lado de otros que son motivos de frustraciones y barreras. Asimismo, distingue entre buenas prácticas implícitas y explícitas:

A. Las primeras están situadas en la experiencia personal

B. Las segundas pueden ser más públicas y sistematizadas

Coffield y Edgard (2009) proponen una serie de dimensiones sobre las buenas prácticas:

- Contexto: considerado como las peculiaridades organizativas de una propuesta para la enseñanza y el aprendizaje, alumnado participante (procedencia, antecedentes, grado en que la propuesta lo valora y toma en consideración), programa formativo y sus relaciones con contextos organizativos, otros proyectos y profesionales implicados.

- Conocimiento: tipo de antecedentes, valores, conocimientos y capacidades organizativas y profesionales de la práctica y sus relaciones con los presupuestos, conocimientos y capacidades disponibles y vigentes dentro de otros contextos, programas y docentes para quienes pudiera ser provechosa. 
- Currículo: una selección de contenidos y aprendizajes la definen y para qué estudiantes puede ser adecuada.

- Pedagogía: metodologías, modos de hacer propuestas y validaciones de las mismas en relación con las existentes en centros o aulas.

- Evaluación: efectos de la práctica relativos a aprendizajes de los estudiantes.

- Gestión: cómo se ha planificado, secuenciado y evaluado la práctica docente y de qué manera ha sido realizada dentro de los márgenes y requerimientos establecidos por las políticas de la administración.

- Aprendizaje docente: habilidades, creencias y valores de los profesores que han desarrollado la práctica y retos que representan a quienes intenten desarrollarla en sus respectivos contextos y condiciones de trabajo.

- Sociedad: Influencias, relaciones y adecuación de una buena práctica con el mercado laboral y la formación de la ciudadanía.

Estas dimensiones de las buenas prácticas educativas acentúan la importancia del contexto, del currículo, de la pedagogía, de la evaluación, del alumnado y, sobre todo, del profesorado, reflexionando sobre las posibles necesidades de formación y sobre la necesidad de vincular una buena práctica con su contexto social. Subrayan la idea de que una buena práctica no es sólo individual sino que debe difundirse entre los docentes y a sus comunidades de referencia profesional incluyéndose dentro de una cultura democrática. "Una democracia viva necesita un enfoque abierto sobre buenas prácticas, sometidas al control de profesionales reflexivos, sensibles a afrontar las complejidades de identificar y diseminar buenas prácticas sociales y educativas" (Coffield y Edgard, 2008: 389).

\section{Características de la investigación}

\subsection{Tipo de investigación}

La investigación que presentamos es de tipo descriptivo pues su objetivo principal es describir sistemáticamente hechos y características de una población o área de interés de forma objetiva y comprobable.

Nuestra investigación es de tipo encuesta porque, conforme a Cohen y Manion (1985), se ocupa de describir las condiciones existentes, identificar valores estándares con los que se pueden comparar las condiciones y determinar las relaciones entre eventos específicos.

\subsection{Objetivos científicos}

En esta investigación, pretendemos dar respuesta a los siguientes objetivos:

1. Conocer algunos principios pedagógicos, que tienen una presencia importante en el aula de inglés como lengua extranjera, y su frecuencia, de acuerdo con la opinión de los participantes en esta investigación.

2. Realizar un estudio comparativo del rendimiento académico en el área de lengua inglesa del alumnado y los principios pedagógicos utilizados en el aula de lengua inglesa. 


\subsection{Muestra}

La muestra es el conjunto de individuos representativos de la población objeto de estudio conforme a un procedimiento específico y reunidos como representación válida del mismo.

En esta investigación, la muestra invitada está compuesta por los 98 alumnos que cursaban la materia de inglés como lengua extranjera en cuarto de ESO. Esta muestra invitada mermó en una alumna porque abandonó los estudios semanas antes de que la prueba fuera realizada.

Otros tres alumnos no completaron todas y cada una de las pruebas por no asistir a clase los días en los que se realizaron las pruebas. Por esta razón, 94 alumnos, 48 alumnas y 46 alumnos, forman la muestra real.

\subsection{Instrumentos para la recogida de datos}

En esta investigación hemos utilizado cuestionarios y tests. Los primeros ofrecen datos referidos a la metodología empleada en clase de lengua inglesa y a las características del alumnado y su entorno. La mayoría de las cuestiones que se presentan son de elección múltiple para que el alumnado pueda indicar la opción que se ajuste a sus circunstancias. En otras, tiene que expresar su opinión usando una escala graduada del uno al cinco, siendo el uno el valor más negativo y cinco el más positivo.

Para conocer el grado de logro en la consecución de distintas tareas y, de esta manera, obtener una nota global partiendo de unas pruebas homogéneas, se han usado tests de lengua.

\subsubsection{Descripción de las pruebas}

\subsubsection{Tests de Comprensión Escrita (Reading)}

La prueba de comprensión escrita ha sido diseñada teniendo en cuenta los siguientes puntos:

- Objetivo que evalúa: Los alumnos deben leer textos adaptados de diferentes tipos e intencionalidad, mostrar comprensión global y específica de los textos y apreciar su estética y figuras literarias. También deben inferir el significado según el contexto.

- Formato: La prueba consiste en la realización de cinco tareas. Cada una de ellas contiene un texto y diferentes actividades de comprensión.

- Extensión: El número de palabras que contiene la prueba es de alrededor de 600. Cada texto contiene entre 57 y 145 palabras.

- Número de ítems: diecinueve.

- Duración: cuarenta y cinco minutos.

- Evaluación de la prueba: A fin de evaluar la comprensión escrita, hemos empleado una escala graduada, numerada y creciente de uno a cinco. El valor más negativo es el uno y el más positivo el cinco. 


\subsubsection{Expresión Escrita (Writing)}

\section{A) Prueba de Expresión Escrita}

La prueba de comprensión escrita ha sido diseñada teniendo en cuenta los siguientes puntos.

- Objetivo que evalúa: El alumnado debe producir, de forma eficaz, distintos tipos de textos con diversas finalidades, en situaciones pasadas, habituales o futuras de comunicación utilizando tanto recursos lingüísticos como no lingüísticos.

- Formato: La prueba consta de diez tareas.

- Extensión de los textos: El número de palabras que el alumno debe producir nunca excede de las 100 palabras en cada tarea.

- Número de preguntas: En total, son 31.

- Duración: Cincuenta y cinco minutos.

- Evaluación de la prueba: Los parámetros evaluados en cada tarea se califican en una escala graduada creciente de uno a cinco, siendo uno el valor más negativo y cinco el más positivo

\section{B) Características de tarea de expresión escrita Project}

- Objetivo que evalúa: Transferir a la lengua extranjera las estrategias de comunicación adquiridas en la lengua materna y utilizar estrategias de aprendizaje y recursos didácticos para narrar acontecimientos.

- Formato: Presentada una cuestión el alumno debe resolverla.

- Tipo de texto: Texto descriptivo y narrativo.

- Actividad: El alumno debe describir o narrar acontecimientos relevantes para el mundo o para su país ocurridos en la década en la que nació. Para documentarse, puede utilizar recursos didácticos como enciclopedias, libros de historia, Internet etc.

- Número de preguntas: Una única cuestión.

\subsubsection{Test de Comprensión Oral (Listening)}

La prueba de comprensión oral ha sido diseñada teniendo en cuenta los siguientes puntos:

- Objetivo que evalúa: El alumnado debe escuchar textos adaptados con diferente intencionalidad y estructura con el objeto de mostrar comprensión tanto de los aspectos generales como específicos y apreciar su estética, además de inferir el significado gracias al contexto. 
- Formato: La prueba consta de seis tareas. Cada una de ellas contiene un texto oral y cuestiones relativas a lo escuchado. Una misma tarea puede incluir más de un texto corto relacionados entre sí conforme a la temática.

- Extensión de los textos: El número de palabras de la prueba es de 643 palabras. Los textos varían de extensión según el tipo de tarea propuesta, entre 26, el más corto, a 179 el más largo.

- Número de preguntas: El número total de ítems es de 12.

- Duración: Cuarenta minutos.

- Evaluación de la prueba: Hemos utilizado una escala graduada creciente de uno a cinco, siendo uno el valor más negativo y cinco el más positivo en cada uno de los parámetros de cada tarea.

\subsubsection{Test de Expresión Oral (Speaking)}

La prueba de expresión oral ha sido diseñada teniendo en cuenta los siguientes puntos:

- Objetivo que evalúa: Los alumnos deberán responder a una serie de cuestiones que expresen situaciones habituales de comunicación utilizando distintos recursos.

- Formato: La prueba consiste en nueve tareas. La extensión de cada una de ellas es distinta. La más corta tiene una sola cuestión, mientras que las más largas incluyen cuatro cuestiones.

- Extensión de los textos: La extensión de los textos varía según el tipo y cantidad de cuestiones en cada una de ellas. Cada texto puede contener entre 40 y 100 palabras aproximadamente.

- Número de Preguntas: El número de preguntas es de veinte en total.

- Duración: La duración de la prueba oscila entre los quince y los veinte minutos aproximadamente.

- Evaluación de la prueba: Para la evaluación de esta prueba hemos utilizado una escala graduada, creciente y numerada del uno al cinco. El uno representa el valor más negativo y el cinco el valor más alto

\subsection{Validez y fiabilidad}

La validez de la pruebas se apoya en el juicio de expertos y por la prueba piloto. A fin de determinar la fiabilidad de nuestras pruebas, hemos aplicado el Modelo Alpha de Cronbach, en aquellas pruebas donde ha sido posible, por haber utilizado una escala para evaluar.

\subsection{Estudio estadístico}

Para el estudio estadístico, hemos utilizado la versión 14.0 del programa SPSS (Statistical Package for the Social Science) para Windows. 


\section{Buenas prácticas educativas para la enSEÑanza Y el aprendizaje del INGLÉS: ASPECTOS PEDAGÓGICOS}

\section{A. El profesor imparte toda la clase en inglés}

Tabla 1. Nota Media en Inglés * El profesor imparte toda la clase en inglés

\begin{tabular}{|l|c|c|c|}
\hline El profesor imparte toda la clase en inglés & Media & N & Desv. típ. \\
\hline Nunca & 2,44 & 16 & 1,413 \\
\hline Pocas veces & 2,00 & 29 & 1,134 \\
\hline Algunas veces & 2,19 & 27 & 1,001 \\
\hline Bastantes veces & 2,40 & 15 & 1,056 \\
\hline Muchas veces & 1,57 & 7 &, 535 \\
\hline Total & 2,16 & 94 & 1,110 \\
\hline
\end{tabular}

Las calificaciones más altas las alcanzan aquel alumnado que afirma que el profesor nunca (2'44) imparte toda la clase en inglés o que lo hace bastantes veces $\left(2^{\prime} 40\right)$, si bien existe poca diferencia entre estas dos opciones. Las peores calificaciones las obtienen aquel alumnado que piensa que la aserción el profesor imparte toda la clase en inglés ocurre muchas veces en el aula. Esto se podría deber a que el nivel de inglés de este alumnado no es muy alto y, por lo tanto, piense que esta circunstancia se da con demasiada frecuencia o con más frecuencia de la deseada por él.

La mayor parte del alumnado, un 59'57\%, declara que la afirmación el profesor imparte toda la clase en inglés se da pocas veces o algunas veces en el aula.

De estos datos, podemos concluir que el impartir toda la clase en inglés no es factor determinante en el rendimiento académico del alumnado en cuarto de Educación Secundaria Obligatoria. Quizás impartir absolutamente toda la clase en inglés no es adecuado en este nivel educativo, cuando la capacidad de la mayoría del alumnado todavía no es muy alta. Esto incluso puede aumentar las inseguridades del estudiante, por ejemplo, para plantear o resolver dudas, obstaculizando la utilización de ciertas estrategias de aprendizaje como la petición de ayuda al profesorado.

\section{B. El profesor nos anima a comunicarnos en inglés}

Tabla 2. Nota Media en Inglés * El profesor nos anima a comunicarnos en inglés

\begin{tabular}{|l|c|c|c|}
\hline $\begin{array}{l}\text { El profesor nos anima a comunicarnos } \\
\text { en inglés }\end{array}$ & Media & N & Desv. típ. \\
\hline Nunca & 1,70 & 10 &, 483 \\
\hline Pocas veces & 2,53 & 19 & 1,307 \\
\hline Algunas veces & 2,14 & 29 & 1,356 \\
\hline Bastantes veces & 2,00 & 20 &, 725 \\
\hline Muchas veces & 2,25 & 16 & 1,000 \\
\hline Total & 2,16 & 94 & 1,110 \\
\hline
\end{tabular}


A pesar de que un 52'12\% del alumnado piensa que el profesor los anima a comunicarse en inglés algunas o bastantes veces en el aula, el alumnado que logra mejores resultados es aquel que piensa que esta situación se da pocas veces en el aula. Este alumnado obtiene una puntuación de 2'53. Un 2'25 consigue el alumnado que cree que se da muchas veces. Los peores resultados los logran aquellos que afirman que el profesor nunca los anima a comunicarse en inglés.

No parece haber una correlación entre el rendimiento académico del alumnado y el hecho de que el profesor anime al alumnado a comunicarse en inglés. No obstante, los estudiantes con mejores resultados piensan que esta circunstancia se da con poca frecuencia en el aula. Quizás el profesor no necesite animar explícitamente a este alumnado porque ya posee una motivación intrínseca lo suficientemente alta para llevar a cabo la tarea con éxito.

\section{En clase de inglés usamos medios audiovisuales}

Tabla 3. Nota Media en Inglés * En clase de inglés usamos medios audiovisuales

\begin{tabular}{|l|r|r|r|}
\hline $\begin{array}{l}\text { En clase de inglés usamos medios } \\
\text { audiovisuales }\end{array}$ & Media & N & Desv. típ. \\
\hline Nunca & 2,31 & 75 & 1,162 \\
\hline Pocas veces & 1,42 & 12 &, 669 \\
\hline Algunas veces & 1,80 & 5 &, 447 \\
\hline Bastantes veces & 2,00 & 1 & - \\
\hline Muchas veces & 2,00 & 1 & - \\
\hline Total & 2,16 & 94 & 1,110 \\
\hline
\end{tabular}

Una gran mayoría de los participantes en esta investigación, concretamente un $79^{\prime} 78 \%$, declara que en clase de inglés nunca usan medios audiovisuales. Este alumnado es el que mayor puntuación consigue con un 2'31. Los dos siguientes grupos en puntuación, ambos con $2^{\prime} 00$, son los que opinan que esta circunstancia se da bastantes o muchas veces en clase de inglés.

No existe una correlación entre el uso de medios audiovisuales en clase y el rendimiento académico del alumnado en inglés. Sin embargo, conviene detenernos en la aseveración de la mayoría de los estudiantes, para reflexionar sobre la dotación de materiales audiovisuales disponibles para el profesorado de lenguas extranjeras y sobre la masificación de los centros educativos que, en muchos casos, obliga a utilizar las aulas específicas, por ejemplo, las de informática como aulas ordinarias, impidiendo el acceso al resto del alumnado. 


\section{En clase de inglés usamos materiales impresos editados}

Tabla 4. Nota Media en Inglés * En clase de inglés usamos materiales impresos editados

\begin{tabular}{|l|c|c|c|}
\hline $\begin{array}{l}\text { En clase de inglés usamos materiales } \\
\text { impresos editados }\end{array}$ & Media & N & Desv. típ. \\
\hline Nunca & 2,38 & 16 & 1,204 \\
\hline Pocas veces & 1,93 & 27 & 1,174 \\
\hline Algunas veces & 2,22 & 36 & 1,072 \\
\hline Bastantes veces & 2,40 & 5 &, 894 \\
\hline Muchas veces & 2,10 & 10 & 1,101 \\
\hline Total & 2,16 & 94 & 1,110 \\
\hline
\end{tabular}

Un 38'29\% piensa que en clase de inglés usan algunas veces materiales impresos editados. Este alumnado logra un 2'22 de media. Las mejores calificaciones las obtienen aquel alumnado que estima que esta situación se da bastantes veces en el aula. Su puntuación es de $2^{\prime} 40$.

Aunque no existe una correlación directamente proporcional entre el uso de materiales impresos editados en clase de inglés y la calificación media del alumnado, si debemos valorar que la mejor calificación media la logra aquel grupo que lo hace bastantes veces.

Según la opinión del alumnado, la mayoría del profesorado de inglés en Educación Secundaria utiliza con cierta frecuencia los materiales impresos editados. Estos materiales proporcionan ventajas al profesorado. Son materiales tradicionales, reutilizables y fácilmente accesibles para el profesorado ya que en prácticamente todos los departamentos de idiomas y / o bibliotecas escolares existen libros de lectura graduados, diccionarios, pósters, tablas, etc.

\section{E. En clase de inglés usamos el casete/cd}

Tabla 5. Nota Media en Inglés * En clase de inglés usamos el casete/cd

\begin{tabular}{|l|c|c|c|}
\hline En clase de inglés usamos el casete/cd & Media & N & Desv. típ. \\
\hline Nunca & 2,00 & 2 &, 000 \\
\hline Pocas veces & 1,75 & 4 & 1,500 \\
\hline Algunas veces & 1,69 & 29 &, 806 \\
\hline Bastantes veces & 2,31 & 39 & 1,055 \\
\hline Muchas veces & 2,65 & 20 & 1,348 \\
\hline Total & 2,16 & 94 & 1,110 \\
\hline
\end{tabular}

La puntuación más alta, un 2'65, la obtiene aquel alumnado que cree que en clase de inglés se usa muchas veces el casete/cd. Este alumnado representa el $21^{\prime} 27 \%$ de 
los participantes. La segunda mejor puntuación, 2'31, la logra los estudiantes que consideran que este hecho se da en clase de inglés bastantes veces. Este segundo grupo está compuesto por el 41'48\% del alumnado participante.

Según los datos, el uso frecuente del casete/cd en clase de inglés repercute positivamente en el logro de resultados más altos. El hecho de que se utilice el casete/cd en clase de inglés en vez de otros soportes electrónicos como televisión, ordenadores, etc. es que los primeros ofrecen ciertas ventajas como: son fáciles de transportar de un aula a otro, son económicos a la hora de ser adquiridos por el centro, el coste de su mantenimiento es mínimo y las editoriales ofrecen cds de audio para explotar las actividades de comprensión auditiva.

\section{F. En clase de inglés usamos el libro de texto}

Tabla 6. Nota Media en Inglés * En clase de inglés usamos libro de texto

\begin{tabular}{|l|c|c|c|}
\hline En clase de inglés usamos libro de texto & Media & N & Desv. típ. \\
\hline Nunca & 2,00 & 1 & - \\
\hline Algunas veces & 1,50 & 2 &, 707 \\
\hline Bastantes veces & 1,44 & 9 &, 527 \\
\hline Muchas veces & 2,26 & 82 & 1,142 \\
\hline Total & 2,16 & 94 & 1,110 \\
\hline
\end{tabular}

Un $87^{\prime} 23 \%$ del alumnado piensa que en clase de inglés se utiliza el libro de texto muchas veces. La calificación media de este alumnado es la más alta. Alcanza el 2'26. De estos datos, podemos deducir que el uso frecuente del libro de texto aumenta el rendimiento académico del alumnado.

Las políticas educativas favorecen que se utilicen los libros de texto en el aula, por ejemplo, la gratuidad de los mismos en centros públicos y concertados en prácticamente todas las comunidades autónomas. Además, el libro de texto ofrece la posibilidad de manejar un corpus de información establecido, muy útil por servir de guía tanto al profesorado como al alumnado.

\section{G. El profesor de inglés manda deberes}

Tabla 7. Nota Media en Inglés * El profesor de inglés manda deberes

\begin{tabular}{|l|c|c|c|}
\hline El profesor de inglés manda deberes & Media & N & Desv. típ. \\
\hline Nunca & 2,00 & 1 & - \\
\hline Pocas veces & 3,25 & 4 & 1,258 \\
\hline Algunas veces & 2,29 & 14 & 1,267 \\
\hline Bastantes veces & 1,96 & 23 &, 976 \\
\hline Muchas veces & 2,13 & 52 & 1,103 \\
\hline Total & 2,16 & 94 & 1,110 \\
\hline
\end{tabular}


La calificación media más alta la logran aquellos alumnos que consideran que el profesor de inglés manda pocas veces deberes. Este grupo de alumnos representa el $4^{\prime} 25 \%$ del total de participantes aunque logran una calificación bastante alta, un 3'25. La segunda mejor media, 2'29, la consiguen los alumnos que piensan que el profesor manda algunas veces deberes de inglés. No obstante, la mayoría, un $55^{\prime} 31 \%$, estiman que esta situación se da muchas veces en el aula. La nota media de este alumnado es notablemente más baja, 2'13.

Estos datos quizás se deban a que el alumnado que es más hábil en el aprendizaje de la lengua inglesa realiza con mejor predisposición sus deberes y, probablemente, los termine antes.

A tenor de estos datos, podemos afirmar que el mandar deberes con una frecuencia media favorece el aprendizaje de la lengua inglesa.

\section{H. Tiempo dedicado a los deberes}

Tabla 8. Nota Media en Inglés * Tiempo dedicado a los deberes

\begin{tabular}{|l|c|c|c|}
\hline Tiempo dedicado a los deberes & Media & N & Desv. típ. \\
\hline 10 minutos o menos & 2,80 & 15 & 1,424 \\
\hline 15 ó 20 minutos & 2,28 & 18 & 1,320 \\
\hline 25 ó 30 minutos & 2,07 & 44 &, 950 \\
\hline 35 ó 40 minutos & 1,67 & 3 &, 577 \\
\hline De 45 minutos a 1 hora & 1,71 & 14 &, 726 \\
\hline Total & 2,16 & 94 & 1,110 \\
\hline
\end{tabular}

En cuanto al tiempo dedicado a los deberes, los mejores resultados los logran el grupo de alumnos que los realizan en diez minutos o menos. Éstos consiguen un 2'80 de media y representan al $16 \%$ de los participantes. A este grupo, le siguen aquellos que hacen sus deberes de inglés entre quince y veinte minutos. Son el 19\% del total y alcanzan el 2'28 de media. Sin embargo, el grupo más numeroso, un $46^{\prime} 80 \%$, dicen dedicarle entre veinticinco a treinta minutos. Su calificación media es más baja, un 2'07.

Estos datos son concluyentes. Los alumnos que tardan menos tiempo en realizar sus deberes son los que mejores resultados alcanzan. Esto se puede deber a que el desarrollo de sus capacidades para hacer los deberes de inglés es mayor que la del resto de sus compañeros y, por este motivo, son más rápidos. Por otra parte, el alumnado que planifica y controla el tiempo dedicado a cada actividad, rinde más y obtiene mayores logros. Así pues, la aplicación de técnicas de estudio favorece el desarrollo de rendimientos académicos más altos. 


\section{Conclusiones}

Conforme a los resultados anteriormente expuestos, podemos extraer las siguientes conclusiones en relación a la consecución de los objetivos que nos hemos propuesto en nuestra investigación.

1. Conocer algunos principios pedagógicos, que tienen una presencia importante en el aula de inglés, y su frecuencia, de acuerdo con la opinión de los participantes en esta investigación.

Con respecto a la afirmación el profesor imparte toda la clase en inglés, un $59^{\prime} 5 \%$ opina que el profesor imparte toda la clase en inglés pocas veces o algunas veces. Aunque lo deseable sería que el profesorado de inglés impartiera casi toda o toda la clase en el idioma objeto, debemos pensar, también, que un profesor debe ajustarse al nivel de competencia lingüística de su alumnado, en este caso, de cuarto de ESO y que el nivel de estos estudiantes es bajo, a tenor de los resultados del informe PISA y las pruebas de diagnóstico de las distintas comunidades autónomas. Además, todavía quedan, en nuestras aulas de inglés como lengua extranjera, algunos residuos de una metodología tradicional basada en la enseñanza de aspectos gramaticales, en detrimento de un enfoque comunicativo en el marco del interaccionismo social como teoría de la adquisición y del socioconstructivimo interaccionista, método ideal en la enseñanza de lenguas extranjeras, según Martín (2009).

Un 30\% afirma que el profesor nos anima a comunicarnos en inglés algunas veces. Probablemente, esta opción, la más votada por el alumnado, refleje solamente aquellas situaciones en que las que el profesorado anima explícitamente a comunicarse en lengua inglesa, pasando desapercibidos otros tipos de estímulos para fomentar la comunicación en la lengua objeto.

En cuanto a la afirmación, en clase de inglés usamos medios audiovisuales, el $79^{\prime} 78 \%$ del alumnado confiesa que en clase de inglés nunca usa medios audiovisuales. Parece impactante esta declaración, más aún en una sociedad TIC como la nuestra. Creemos que, tras este porcentaje, subyace una crítica sobre la dotación de medios audiovisuales en los centros públicos y concertados de educación secundaria, así como el acceso a los mismos por parte del profesorado de lenguas extranjeras. Paralelamente, los estudiantes confiesan que en clase de inglés usan el casete/cd bastantes veces (un 41'50\% de los estudiantes); usan el libro de texto muchas veces (un 87'24\%) y que usan materiales impresos editados (un 38'30\%) algunas veces. Estos soportes y materiales son una alternativa más económica que los medios audiovisuales, exigen un menor mantenimiento y son más accesibles para el profesorado de lenguas extranjeras.

Finalmente, la mayoría del alumnado, un 55'32\% opina que el profesor manda deberes. El tiempo dedicado a los mismos, es de entre 25 minutos a 30 minutos para el $46^{\prime} 80 \%$. Este tiempo parece excesivo y, quizás, denote una falta de planificación del tiempo dedicado a la realización de tareas por parte de este alumnado, a diferencia de sus compañeros que los realizan en diez minutos o menos y, cuyas calificaciones en inglés son las más altas. 
2. Realizar un estudio comparativo del rendimiento académico en el área de lengua inglesa del alumnado y los principios pedagógicos utilizados en el aula de lengua inglesa.

De esta investigación se desprende que existen ciertas prácticas pedagógicas que favorecen el aprendizaje de la lengua inglesa. Así, el alumnado que afirma que las siguientes prácticas se dan en su clase de inglés obtiene mejores calificaciones: el uso frecuente de materiales impresos editados, el uso frecuente del casete/cd, la utilización del libro de texto con mucha frecuencia y el que el profesor mande deberes para casa con una baja frecuencia y que la duración de los mismos sea de unos diez minutos.

Otras prácticas, sin embargo, no parecen tener una especial relevancia en la adquisición de la lengua inglesa: el que el profesor imparta toda la clase en inglés, el que el profesor siempre anime a que su alumnado se comunique en inglés y el uso de medios audiovisuales.

\section{REFERENCIAS BIBLIOGRÁFICAS}

ABDOULAYE, A. (2003). Conceptualisation de "bonnes pratiques" en education: essai d'une approche internationale à partir d'enseignements tirés d'un projet. En Développment curriculaire et "bonne pratique" en éducation. Ginebra: Bureau International d'Éducation. Disponible en: www.ibe.unesco.org. [Consulta: 2810, 2011].

ALEXANDER, R. (1997). Policy and Practice in Primary Education: Local Initiative, Nacional Agenda. London: Routledge.

BOLÍVAR, A. (1999). Cómo Mejorar los Centros Educativos. Madrid: Síntesis.

BRANNAN, T.; DUROSE, C.; JOHN, P. y WOLMAN H. (2006). Assessing Best Practices as a Means of Innovation. Paper presented at the Annual Conference of the Urban Affair Association. Montreal. Canadá. (22 April). Disponible en: http://www.ipeg.org/uk/publications/index.php. [Consulta: 28-10, 2011].

COHEN, L. y MANION, L. (1990). Métodos de Investigación Educativa. Madrid: La Muralla.

COFFIELD, F. y EDWARD, S. H. (2009). Rolling out "good", "best" and "excellent" practice. What next? Perfect Practice? British Educational Research Journal, 35 (3), 371-390.

COLÁS, M. P. y BUENDÍA, E. (1994). Investigación Educativa. Sevilla: Alfar.

CORPAS, M. D. (2012). Multiculturalidad y Publicidad en Educación Secundaria: Un proyecto Educativo Europeo. En L. Luque Nadal (Ed.), Transculturalidad, Lenguaje e Integración, pp. 149-160.

ESCUDERO, J. M. (2009). Buenas prácticas y programas extraordinarios de atención al alumnado en riesgo de exclusión educativa. Profesorado, 13 (3), 107-141.

HARGREAVES, A. (2003). Enseñar en la Sociedad del Conocimiento. Barcelona: Octaedro.

MARTíN, M. A. (2009). Historia de la metodología de enseñanza de lenguas extranjeras. Tejuelo, 5, 54-70. 
MCMILLAN, J. y SCHUMACHER, S. (2005). Investigación educativa. Madrid: Pearson Educación.

PHIPPS, A. y GUILHERME, M. (2004). Modern Languages: Learning and Teaching in an Intercultural Field. London: Sage.

SÁNCHEZ CARRIÓN, J. J. (1999). Manual de análisis estadístico de los datos. Madrid: Alianza Editorial.

SHOHAMY, E. (2006). Language Policy: Hidden Agendas and New Approaches. London: Routledge.

WILLIS, J. (2002). Teaching English through English: a course in classroom language and techniques. London: Longman. 
